\title{
Land use uncertainty in transportation forecast
}

\author{
Hana Ševčíková \\ Puget Sound Regional Council \& University of Washington \\ HSevcikova@psrc.org \\ Brice Nichols \\ Puget Sound Regional Council \\ BNichols@psrc.org
}

\begin{abstract}
Using an integrated land use and travel model system implemented for the Puget Sound region in Washington state, a Bayesian Melding technique is applied to represent variations in land use outcomes, and is propagated into travel choices across a multi-year agent-based simulation. A scenario is considered where zoned capacity is increased around light rail stations. Samples are drawn from the posterior distribution of households to generate travel model inputs. They allow for propagation of land use uncertainty into travel choices, which are themselves assessed for uncertainty by comparing against observed data. Resulting travel measures of zonal vehicle miles traveled (VMT) per capita and light rail station boardings indicate the importance of comparing distributions rather than point forecasts. Results suggest decreased VMT per capita in zones near light rail stations and increased boardings at certain stations with existing development, and less significant impacts around stations with lower initial development capacity. In many cases, individual point level comparisons of scenarios would lead to very different conclusions. Altogether, this finding adds to a line of work demonstrating the policy value of incorporating uncertainty in integrated models and provides a method for assessing these variations in a systematic way.
\end{abstract}

Article History:

Received: July 20, 2020

Revised: March 17,

2021

Accepted: March 18, 2021

Available online: July

26,2021

\section{Introduction}

Long-range land use and transportation planning is a critical focus area for regions across the world. Despite the many unknowns of upcoming decades, forecasting can provide some context for optimal resource allocation to guide urban growth. In typical applications, point forecasts are generated for specific policy scenarios or project sets to form a relative comparison of costs and benefits. Single values that represent unique scenarios are easily digestible for policy analysis, but the planning community has long been aware of the limitations and concerns of relying on such simplifications given a pipeline

Copyright 2021 Hana Ševčíková and Brice Nichols.

doi: $10.5198 /$ jtlu.2021.1853

ISSN: 1938-7849 | Licensed under the Creative Commons Attribution - NonCommercial License 4.0.

The Journal of Transport and Land Use is the official journal of the World Society for Transport and Land Use (WSTLUR) and is published and sponsored by the University of Minnesota Center for Transportation Studies. 
of uncertainties around input data and model limitations (de Jong et al. 2005; Flyvbjerg 2005; Flyvbjerg et al. 2003, 2005; Hartgen 2013; Hugosson 2005; Salling and Banister 2009). The uncertainties of travel models (Conway et al. 2018; de Jong et al. 2005; Sumalee et al. 2009) and land use models (Pontius Jr and Spencer 2005; Ševčíková et al. 2007; Tayman 1996, 2009, 2011) have been examined both separately, and to a lesser degree in conjunction (Clay and Johnston 2006; Duthie et al. 2010; Kockelman 2002, 2003; Pradhan and Kockelman 2002; Rodier 2005; Ševčíková et al. 2011; Zhao and Kockelman 2002). Wang and Kockelman (2018) give a review of uncertainty methods in integrated land use transportation models.

This study seeks to continue this line of research by using current state-of-the-practice models and policy scenarios to quantify uncertainties in complex integrated agent-based models. As the regional metropolitan planning organization for the Puget Sound region of the U.S., the Puget Sound Regional Council (PSRC) develops long-range land use and transportation policies to manage growth through 2040 and beyond. Adopted land use plans aim to concentrate new housing, jobs, and development to regional growth centers, which are strategic nodes supported by high capacity transit. A regional light rail, commuter rail, and bus-rapid-transit network is currently being planned and expanded to support these land use policies. As policy updates are now being considered to 2050, the specific distribution of housing and job growth is under discussion, with decisions being informed from results of integrated land use and travel model simulations.

Differentiating between scenarios is important to understand the magnitude of impacts policies have in directing growth. However, differences in point forecasts without taking account of uncertainty can lead to wrong conclusions. Instead, deriving differences between whole distributions of the particular scenarios gives a more informed basis for robust policy decisions.

In this paper, we consider a hypothetical sensitivity test of growth allocation to light rail stations via zoning changes to allow denser development. In particular, we double the allowed residential and nonresidential density in areas around current and future light rail stations. The goal is to make a robust comparison between the scenario and the baseline under the consideration of uncertainty. We assess uncertainty in the land use model forecast using the Bayesian Melding method (Ševčíková et al. 2011) and propagate it into travel model outcome measures of vehicle miles traveled and light rail boardings. As a result, we obtain a posterior distribution of differences between the scenario and the baseline. Propagating land use uncertainty derived via an established statistical method into an activity-based travel model operating on very disaggregated level of geography, namely parcel, is a novel approach. Note that the scenario considered here is taken as a rather extreme policy compared to actual proposed alternatives and is considered only to illustrate the methods and ideas presented here, not as an official representation of policy. Importantly though, the framework does represent a model system that is currently in use, and thus provides some insight into how an analysis of uncertainty might be applied to a real-world type of analysis.

The paper is organized as follows. Section 2 describes our integrated land use and travel models, (known as UrbanSim and SoundCast, respectively). Section 3 reviews the methodology and describes findings from prior studies. Section 4 contains the core of this paper. It describes our application, including the data and method we use, followed by the results. Section 5 concludes the paper and gives suggestions how we plan to extend the study to gain more insights into applications of this type.

\section{Integrated Land Use and Travel Model}

The modeling framework used for this work is an integrated land use model, UrbanSim, combined with a travel model, SoundCast. PSRC employs these models for long-range forecasting and scenario planning purposes for the greater Seattle region with forecast horizons of 30 or more years. 


\subsection{UrbanSim}

UrbanSim (Waddell 2002) is an urban simulation model operational in several urban areas in the United States (Waddell 2002, 2007, 2010, 2011) and Europe (Felsenstein et al. 2010). The system is implemented as a set of interacting agent-based models that represent the major actors and choices in the urban system, including households choosing residential locations, business choices of employment location, worker choices of jobs, and developer choices of locations and types of real estate development. The model system microsimulates the annual evolution in locations of individual households and jobs, including the connection between them, and the evolution of the real estate within each individual geography as the result of actions by real estate developers. It uses regional control totals for number of households and jobs obtained from external sources.

We use a version of UrbanSim that is a part of the OPUS framework, or Open Platform for Urban Simulation (Waddell et al. 2005, 2015), whose modular structure allows the application to be easily adapted to regional conditions. The models are summarized in Table 1.

Table 1: UrbanSim models in the PSRC application.

\begin{tabular}{|c|c|c|}
\hline Model & Method & Description \\
\hline Real estate price & hedonic regression & Predicts prices of parcels. \\
\hline Expected sale price & hedonic regression & Predicts prices of possible real estate proposals. \\
\hline Development proposal choice & weighted random sampling & $\begin{array}{l}\text { Chooses real estate proposals to be built (including } \\
\text { redevelopment) based on the expected sales prices } \\
\text { of proposals. }\end{array}$ \\
\hline Building construction & rule based & $\begin{array}{l}\text { Demolishes buildings (for redevelopment) and } \\
\text { builds new buildings according to the chosen } \\
\text { proposals. }\end{array}$ \\
\hline Household transition & random sampling & $\begin{array}{l}\text { Creates and removes households. It is driven by } \\
\text { macroeconomic predictions which provide regional } \\
\text { control totals for households grouped by their } \\
\text { characteristics. }\end{array}$ \\
\hline Employment transition & random sampling & $\begin{array}{l}\text { Creates and removes jobs. It is driven by macro- } \\
\text { economic predictions which provide regional control } \\
\text { totals for jobs grouped by employment sectors. }\end{array}$ \\
\hline Household relocation & weighted random sampling & Determines households for moving. \\
\hline Household location choice & $\begin{array}{l}\text { multinomial logit with } \\
\text { random sampling of alternatives }\end{array}$ & Locates moving households into buildings. \\
\hline Employment relocation & weighted random sampling & Determines jobs for moving. \\
\hline Employment location choice & $\begin{array}{l}\text { multinomial logit with } \\
\text { random sampling of alternatives }\end{array}$ & Locates moving jobs into buildings. \\
\hline Work at home choice & binary logit & $\begin{array}{l}\text { Simulates workers decision to work at home or } \\
\text { out of home. }\end{array}$ \\
\hline Workplace relocation & rule based & Simulates workers decision to change job. \\
\hline Workplace choice & $\begin{array}{l}\text { multinomial logit with } \\
\text { random sampling of alternatives }\end{array}$ & Assigns workers to jobs. \\
\hline
\end{tabular}

There are many sources of uncertainty in a model of this type, and the column Method in Table 1 includes some of them, such as stochasticity due to random sampling or errors in estimated parameters in regression and logit models. In addition, imputation and possible biases in the input data or uncertainty in the structure of the models all contribute to the overall uncertainty.

\subsection{SoundCast}

SoundCast is an activity-based travel model that simulates individual travel choices across a typical weekday for all persons and households in the region. Travel choices range from long-term models such as the number of vehicles owned per household to daily decisions such as destination choice, mode choice and route selection for individual trips. The sum of these choices yield travel demand measures such as traffic volumes on roads by time of day, transit boardings, and other measures that can be evaluated and compared to scenarios with changes in land use patterns, transportation networks, accessibilities, and pricing policies. SoundCast choice models use parcel-level input data from UrbanSim 
such as household and job locations in conjunction with roadway network and transit service information to simulate choices that represent a typical workday in the Puget Sound region (Bowman and Bradley 2014). As an activity-based travel model, SoundCast is a state-of-the-practice tool for project evaluation and scenario comparison for regional land use and transportation planning. SoundCast was used to measure project impacts for PSRC's Regional Transportation plan in 2018 (Puget Sound Regional Council 2018) as well as long-range land use planning for PSRC's Vision 2050 process.

As with UrbanSim, SoundCast's models are subject to the same issues of uncertainty stemming from logit model parameter estimation and random sampling used to simulate discrete choices from probability distributions (Castiglione et al. 2015). SoundCast is designed to reduce simulation error by iteratively updating demand choices with network accessibilities until changes stabilize, but for large regional-scale models, perfect convergence is not always practically achievable due to long model run times. Recommended convergence parameters were employed for this test (Gibb and Bowman 2007), as used for production-level scenario analysis, but there is likely still some uncertainty due to this iterative process.

\subsection{UrbanSim $\times$ SoundCast Integration}

Most land use and travel models used for regional planning require some level of integration by definition; land use decisions are often dependent on travel accessibility, while transportation decisions are dictated by the accessibility of nearby activities and opportunities. The models are highly interdependent such that the input of a travel model is the output of a land use model, and vice versa. Aggregate decisions people make as a result of changes in the built environment will directly affect their travel patterns and subsequently affect location decisions and the built environment. For example, a new office development draws traffic, but accessibility may be diminished over time as congestion grows, leading to changes in destination and location decisions.

In reality, these choices are tightly coupled, such that impacts to land use patterns could happen rather quickly, on a model scale of months or years. In practice, the data exchange between land use and travel models is less frequent as used by PSRC. At best, land use accessibility measures are updated around 10 to 15 model years by running the travel model before proceeding with land use forecasts, or perhaps only for a final forecast year. For PSRC, a full SoundCast run requires up to 24 hours to reach an acceptably equilibrated solution for travel demand. In settings required for policy analysis, UrbanSim's run times require up to 24 hours for a 30-year forecast. Together the total run time of a full-scale integrated run consumes at least 4 days (not counting time required to produce and compare results). Given the exceptional growth and myriad of projects scheduled for the region within this planning horizon, it's likely that travel measures should be updated as often as every 5 years, which would bring this type of run to 8 days. Clearly, this is impractical for testing numerous scenarios and responding nimbly to requests from policy makers in context of shaping and comparing scenarios. Even at half this run time, the potential accuracy from tighter model integration is rendered moot by the practical limitations of computer hardware and perhaps less than optimal software design.

Given such constraints, introducing the impacts of uncertainty through repeated simulations of the entire model suite is not feasible. Therefore, understanding uncertainty around outputs for benchmark model years, as explored in this paper, is a useful and practical method for improving insight on relative difference between policy scenarios.

\section{Assessing Uncertainty}

To quantify uncertainty around travel model outcomes, we first assess uncertainty around UrbanSim outputs, using an established statistical methodology described in this section. The following section then shows how such uncertainty can be propagated into SoundCast outputs. 


\subsection{Methodology}

To assess uncertainty around our land use model, we use a method called Bayesian Melding. It was originally developed to assess uncertainty in deterministic models (Poole and Raftery 2000; Raftery et al. 1992, 1995). Ševčíková et al. $(2007,2011)$ extended the method to work with stochastic models and adapted it to an agent-based simulation context. Yang and Guo (2019) applied the method in other areas. Here we briefly review the method. For more details, we refer readers to the above publications.

Figure 1 communicates the basic concept. At the starting point, $t_{0}$, we have data available necessary to run the model. There is a prior distribution of model inputs $q(\Theta)$ from which we draw input values $\Theta_{i}$ for $i=1, \ldots, I$. The model runs $I$ times from the starting point to the present, $t_{1}$, and for each input $\Theta_{i}$ it produces as output the quantity of interest, $\Phi_{i}$. Time $t_{1}$ is defined as a time point for which we have observed data available. We use the observed data, denoted by $y$, to estimate the model bias and run-specific variance, denoted by $a$ and $\sigma_{i}^{2}$, respectively. Furthermore, we compute a weight $w_{i}=L\left(\Phi_{i}\right)$ for each input $\Theta_{i}$, where $L\left(\Phi_{i}\right)$ is the likelihood of the model outputs given the observed data, $L\left(\Phi_{i}\right)=\operatorname{Prob}\left(y \mid \Phi_{i}\right)$. For each of the $I$ runs, the model is run forward until a future time, $t_{2}$, for which we make a prediction. The result is denoted by $\Psi_{i}$. Combining the bias, variance and the weights with model outputs yield the posterior distribution of $\Psi$.

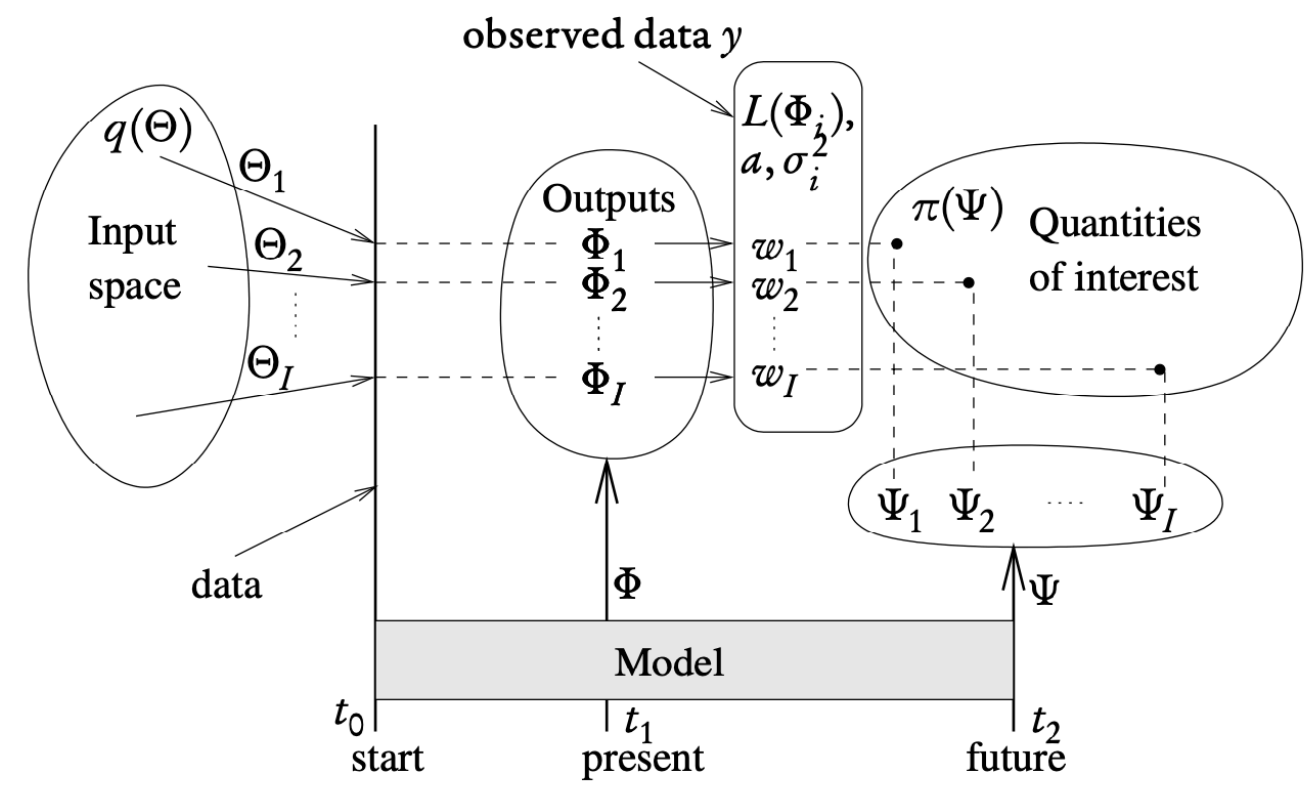

Figure 1: Illustration of the Bayesian Melding method.

The underlying statistical model is defined on the level of geography on which observed data $y$ is available, here indexed by $k$, and for each quantity of interest, indexed by $l$, while $i$ indexes the simulation run. Then the model is defined as

$$
\left(y_{k l} \mid \Theta=\Theta_{i}\right)=\mu_{i k l}+a_{l}+\epsilon_{i k l}, \quad \text { where } \epsilon_{i k l} \stackrel{\text { iid }}{\sim} N\left(0, \sigma_{i l}^{2}\right)
$$

for $i=1, \ldots, I, k=1, \ldots, K$ and $l=1, \ldots, L$. The quantity $\mu_{i k l}$ is the expected value of $y_{k l}$ under the model given $\Theta_{i}, \epsilon_{i k l}$ denotes the model error, and $a_{l}$ is the overall bias in the model predictions of the $l$ th output. We can estimate $\mu_{i k l}$ by the predicted value of $y_{k l}$ which is the model output at $t_{1}$, 
$\Phi_{i k l}$. Then the bias and variance are estimated as

$$
\begin{aligned}
\hat{a}_{l} & =\frac{1}{I K} \sum_{i, l}\left(y_{k l}-\Phi_{i k l}\right), \quad \text { and } \\
\hat{\sigma}_{i l}^{2} & =\frac{1}{K} \sum_{k}\left(y_{k l}-\hat{a}_{l}-\Phi_{i k l}\right)^{2} .
\end{aligned}
$$

This yields a conditional predictive distribution of the quantities of interest:

$$
y_{k l} \mid \Theta_{i} \sim N\left(\hat{a}_{l}+\Phi_{i k l}, \hat{\sigma}_{i l}^{2}\right)
$$

with weights computed as

$$
w_{i} \propto p\left(y \mid \Theta_{i}\right)=\prod_{l=1}^{L} \prod_{k=1}^{K} \frac{1}{\sqrt{2 \pi \hat{\sigma}_{i l}^{2}}} \exp \left[-\frac{1 / 2\left(y_{k l}-\hat{a}_{l}-\Phi_{i k l}\right)^{2}}{\hat{\sigma}_{i l}^{2}}\right] .
$$

The bias, variance and weights in Equations 2, 3, and 5 are estimated at time $t_{1}$. The marginal distribution of the $l$ th quantity of interest, $\Psi_{k l}$, in the future time $t_{2}$ is given by a mixture of normal distributions:

$$
\pi\left(\Psi_{k l}\right)=\sum_{i=1}^{I} w_{i} N\left(f_{l}^{a}\left(\hat{a}_{l}\right)+\Psi_{i k l}, f_{l}^{v}\left(\hat{\sigma}_{i l}^{2}\right)\right), k=1, \ldots, K, l=1, \ldots, L
$$

Functions $f_{l}^{a}, f_{l}^{v}$ determine how the bias and variance of indicator $l$ are propagated over the time period $\left[t_{1}, t_{2}\right]$. This can be done for example by applying propagation factors estimated in prior studies, as was done in Ševčíková et al. (2015).

In order to stabilize the variance in Equation 4, often the model results need to be transformed to a different scale. In cases where the quantity of interest are counts (which is the case of our application), a square root transformation is often a good choice. In such a situation, $\Phi_{i k l}=\sqrt{\Phi_{i k l}^{r}}, \Psi_{i k l}=\sqrt{\Psi_{i k l}^{r}}$ and $y_{k l}=\sqrt{y_{k l}^{r}}$ where $\Phi_{i k l}^{r}$ and $\Psi_{i k l}^{r}$ are the raw model outputs at $t_{1}$ and $t_{2}$, respectively, and $y_{k l}^{r}$ are the raw observed data at $t_{1}$. The marginal distribution $\pi\left(\Psi_{k l}^{r}\right)$ is derived by applying the corresponding inverse transformation to $\pi\left(\Psi_{k l}\right)$.

\subsection{Prior Studies}

In Ševčíková et al. (2007), the method was applied to one land use indicator only, namely the number of households. A posterior distribution of household counts was derived for each of the 295 Traffic Analysis Zones (TAZ) in the Eugene-Springfield, Oregon region and their aggregates. The study compares the Bayesian Melding results to a distribution formed by multiple runs only, i.e. without applying the Bayesian Melding methodology. One of the conclusions is that multiple runs greatly underestimate the uncertainty in models of this type, while Bayesian Melding yield well calibrated results. It shows that simply repeating simulations, even with varying inputs, does not account for all uncertainty. For example, multiple runs would not account for uncertainty due to model structure, while Bayesian Melding does.

While the previous study used a sole land use model, Ševčíková et al. (2011) applied the method to an integrated land use \& travel model framework for the Puget Sound Region, and propagated the land use uncertainty into the travel model outcome of travel time. The question of interest was how do the travel times on selected routes change when a major highway in the region, namely the Alaskan Way Viaduct in Seattle, is removed from the network. A 4-step travel model was used which takes TAZ level inputs on households and jobs by sector. These inputs were sampled from the posterior distribution 
resulting from applying the Bayesian Melding method to the land use model outputs. On the travel model output side, using validation data on travel times for the selected routes, a bias correction was made and the assessed variance was added to the final travel times, yielding a distribution of the travel times for each route. These were then used to derive a distribution of the differences in travel times between the two scenarios.

Ševčíková et al. (2015) shows how Bayesian Melding, developed in an academic environment, has been integrated into an official land-use forecast published by the PSRC. It targets practitioners, such as planners and policy makers, to provide a step-by-step recipe to assess uncertainty about forecasted quantities. Only uncertainty about the land use model is considered.

\section{Uncertainty in Integrated System}

We now demonstrate the assessment of land use uncertainty and its propagation into travel model outcomes using an example. In our application, we seek to assess the impact of a zoning change on travel model outcomes while accounting for uncertainty. More specifically, we compare a base scenario to a scenario in which we double the development capacity around light rail stations. By propagating the land use uncertainty into the activity based travel model, we aim to obtain distributions of vehicle miles traveled and light rail boardings.

Thus, we setup the following scenarios:

Base: The zoning dataset corresponds to the currently adopted future land use layer.

TOD: The zoning dataset is modified so that future structures in light rail TOD (transit oriented development) buffers are allowed to accommodate twice as many households and jobs than the Base zoning allows. In addition, the capacity of current structures in these buffers is doubled.

\subsection{Data}

Our UrbanSim simulation begins in 2014. For this base year, $t_{0}$, we have a detailed (parcel-level) information on households, population, jobs and existing real estate. Our "present" time, $t_{1}$, is 2018, for which we have observed number of households $\left(y_{k}\right)$ on the census block group level, $k=1, \ldots, 2645$. We set the target year, $t_{2}$, to be 2040 .

Our inputs to the travel model include a 2040 network, which has all future light rail stations included. We identified all parcels that are within one mile buffers from these stations, measured via network distance. These are the parcels affected by the TOD scenario where development capacity is increased.

For assessing uncertainty of the travel model itself, we compare observed and modeled values of vehicle miles traveled on the Forecast Analysis Zone (FAZ) level in the base year, as well as boarding counts for all current light rail stations.

\subsection{Method}

The process is depicted in Figure 2. First, we run UrbanSim from 2014 to 2040 ten times $(I=10)$. For each of those runs we varied its inputs in terms of model coefficients, mobility rates and random seed. Following Ševčíková et al. (2007), coefficients and rates were sampled from a normal distribution where the center and spread was determined by the estimated values and the standard error, respectively. Using the observed data $y_{k}$ and modeled outputs $\Phi_{i k}$ at 2018, we obtain $w_{i}$ and $\hat{\sigma}_{i}$ (Equations 5 and 3). Note that in this exercise, we use the number of households as the single quantity of interest. Thus, $L=1$ and we omit the index $l$ for simplicity. Also note that we will condition our results on the external control totals and therefore set the bias a to zero.

Using the model output at 2040, $\Psi_{i k}$, we now have all components we need to construct the posterior distribution for the number of households in each census block group, $\pi\left(\Psi_{k}\right)$ (Equation 6), depicted in Figure 2 by the box plots on the top. 


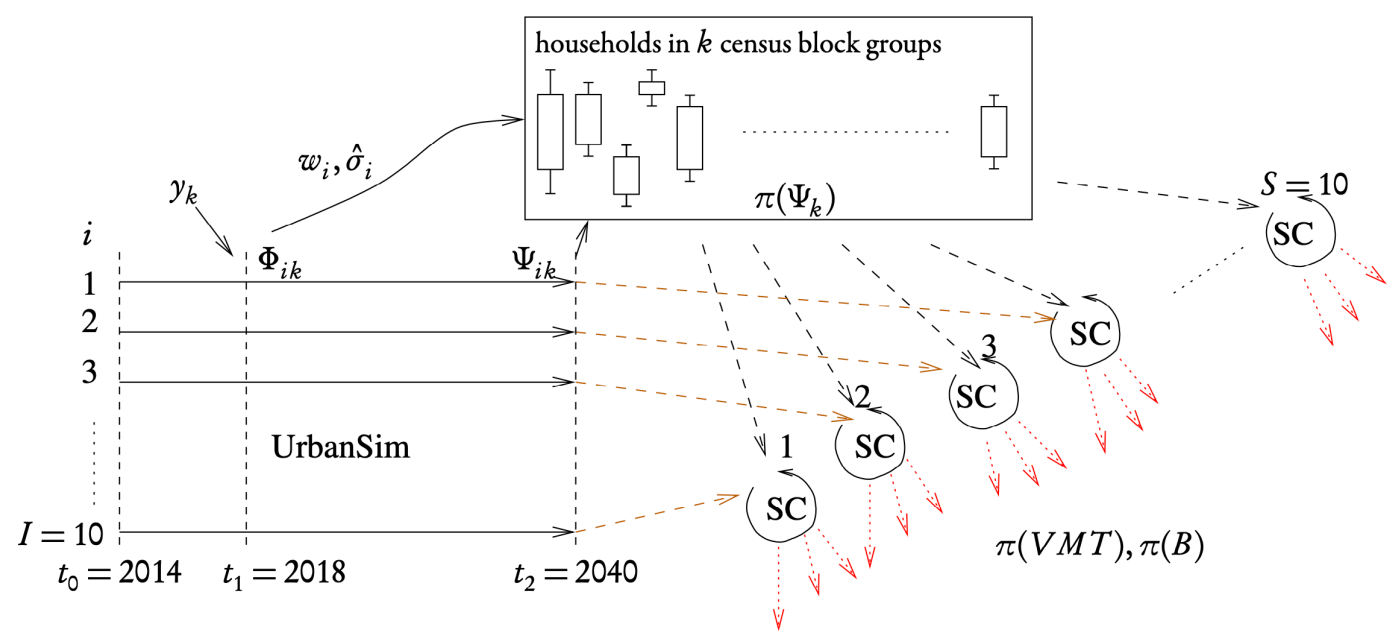

Figure 2: Propagation of Bayesian Melding results into SoundCast.

To prepare land use inputs for SoundCast (SC), we now sample from $\pi\left(\Psi_{k}\right)$, say $\left(\psi_{1}, \ldots, \psi_{K}\right)^{1}, \ldots,\left(\psi_{1}, \ldots, \psi_{K}\right)^{S}$. Each of the black dashed arrows in the figure corresponds to one such set of sampled values (which are scaled to sum to the regional control total) and represents one possible set of land use inputs. There are two additional aspects that need an attention:

1. Since SoundCast operates on the parcel level, the sampled values have to be disaggregated from census block group to parcel. To do this, we randomly couple each of the $S$ samples with one of the $I$ UrbanSim runs and derive corresponding parcel-level scaling factors.

2. The person-jobs relationship established in the workplace choice model of UrbanSim might get invalidated while sampling (households might get removed or new households added). Thus, the workplace choice model is re-run on the coupled UrbanSim runs, using the corresponding sample of number of households.

This post-simulation processing is depicted in the figure by the brown dashed arrows. SoundCast then runs on each of the post-processed samples for 2040 which yields $S$ runs. We use $S=10$ for each scenario.

Using the empirical variance estimated from the observed data of VMT and boarding counts (see below), and using the SoundCast results from the $S$ runs, we can construct a posterior distribution of VMT and boarding counts (red arrows in the figure).

To estimate the empirical variance of VMT, we compare the observed and modeled VMT on the FAZ level in the base year. We find that a square root transformation gives the best fit with regards to constant residuals, see Figure 3. While the bias is negligible, the standard error is 112.1. Thus, the resulting VMT can be written as a vector

$$
\begin{aligned}
V M T_{k} & =\left(V_{1, k}^{2}, V_{2, k}^{2}, \ldots, V_{S, k}^{2}\right) \quad \text { where } \\
V_{s, k} & \sim N\left(\sqrt{\tau_{s, k}}, 112.1^{2}\right) \quad \text { for } s=1, \ldots, S .
\end{aligned}
$$

Here, $\tau_{s, k}$ is the VMT in FAZ $k$ modeled by the $s$-th SoundCast run.

To estimate the empirical variance of light rail boardings, the same procedure was followed. Using our observed and modeled data it results in the standard error of 7.14 on the square root scale.

Having a sample from the multivariate probability distribution of the vector $V M T_{k}$, any function can be applied to it in order to obtain the distribution of other VMT related indicators. When deriving a distribution that includes quantities that vary across SoundCast runs, e.g. land use input, 


\section{VMT on FAZ level}

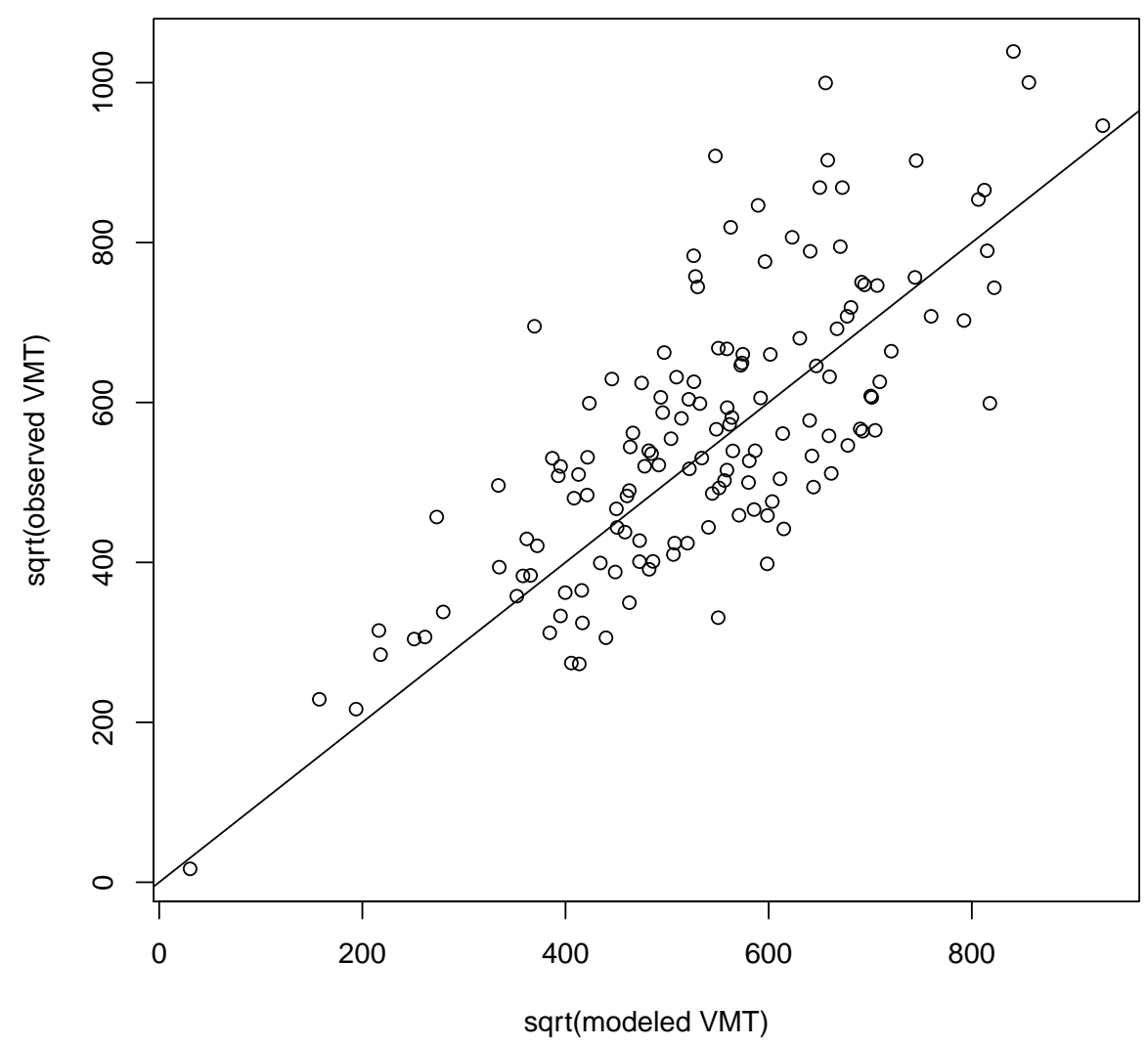

Figure 3: Comparison of observed and modeled VMT. Each dot represents one FAZ. The black line is the $x=y$ line.

the desired function can be applied directly on the $V_{s, k}$ measures. For example, to obtain VMT per capita (VMTC), we divide each sampled VMT value by the FAZ population. Since the population differs in each run $s$, we use

$$
\begin{aligned}
V M T C_{k} & =\left(V C_{1, k}, V C_{2, k}, \ldots, V C_{S, k}\right) \text { where } \\
V C_{s, k} & =V_{s, k}^{2} / P_{s, k} \quad \text { for } s=1, \ldots, S
\end{aligned}
$$

$P_{s, k}$ is the population in FAZ $k$ used as the input for the $s$-th SoundCast run.

In terms of the total time required to process our model setup, with a cluster of 10 nodes, we were able to obtain results in about 27 hours for each scenario. This includes 3 hours for one UrbanSim simulation and 24 hours for one SoundCast run. Intuitively, the time increases linearly with the number of nodes available.

Finally, note that our land use model training period of 4 years (from 2014 to 2018) is rather short, making the model more uncertain, which increases the importance of assessing the uncertainty. When new data become available, the Bayesian melding quantities can be re-estimated to increase their precision. 


\subsection{Results}

\subsubsection{VMT per capita}

Using the method from the previous section, we obtain the predictive distribution for the VMT per capita for each of the 219 FAZes (Equation 8). In Figure 4, results are shown for the ten largest FAZes measured on population. While the raw model results from the $S$ runs are shown as black dots, the distribution is summarized into $50 \%$ and $80 \%$ probability intervals depicted as whiskers and end lines, respectively.

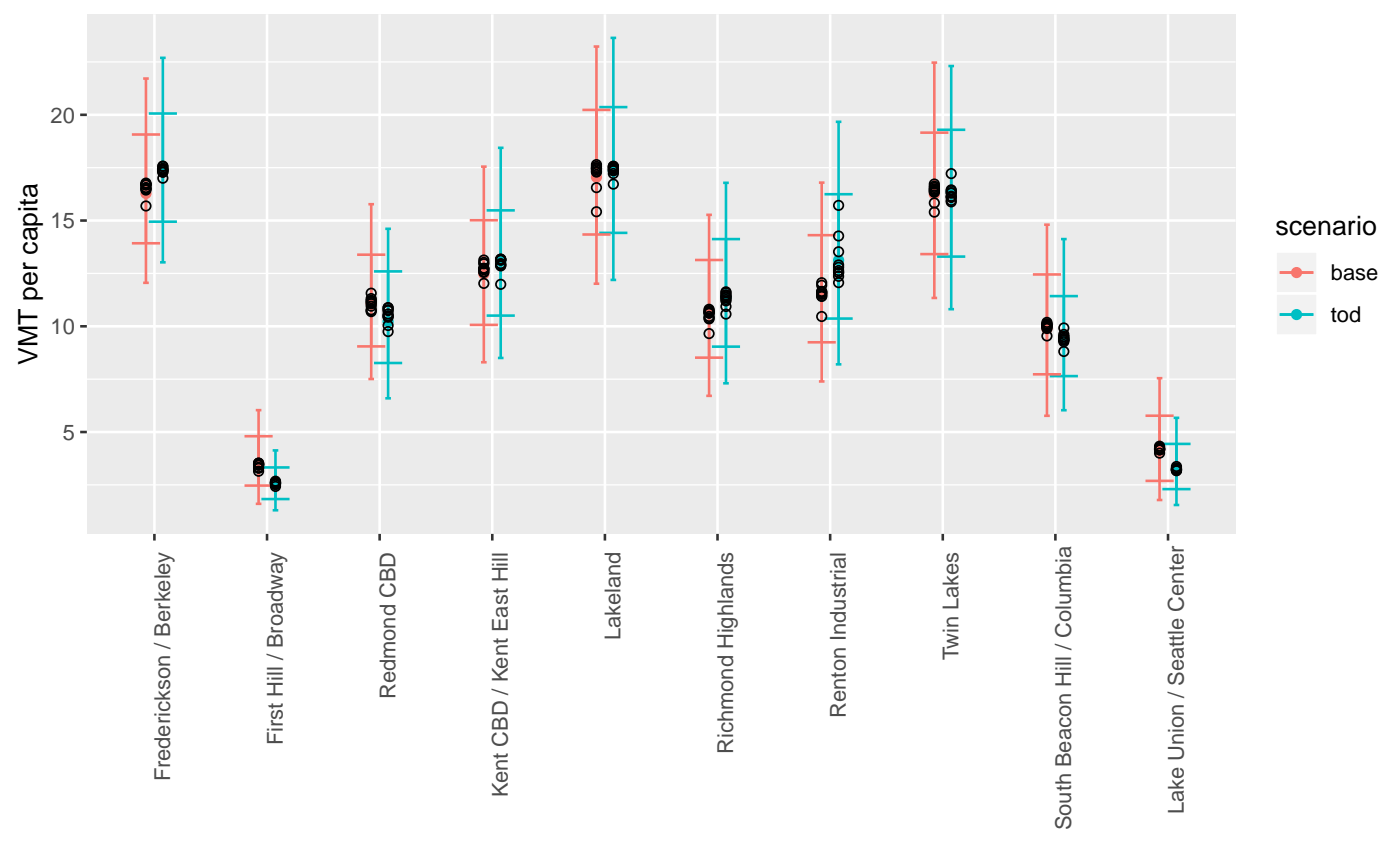

Figure 4: Predictive distributions of VMT per capita for 10 largest FAZes in the region for the two scenarios, base (red) and tod (blue). The 50\% probability intervals are marked by the whiskers, while the line ends mark the $80 \%$ probability intervals. The black dots show the point results from the model.

One of the main goals of such analysis is to determine the location and extent of any significant variations between the two scenarios. In usual point prediction settings, we would compare a single dot from each scenario to one another. As can be seen in Figure 4, such comparison can lead to conclusions that are strongly dependent on the specific runs. For example, consider the Renton Industrial FAZ. If we would compare the Base run that results in the lowest dot on the red line with the TOD run that results in the highest dot on the blue line, we would conclude that there is an increase of more than 5 VMT per capita, which is an increase of more than $50 \%$ for this FAZ. However, if our two comparing runs are the ones resulting in dots much closer together, the conclusion would suggest almost no difference between the two scenarios. Adding uncertainty information helps in providing more robust comparisons. The difference between the medians of the two distributions is 1.6, which corresponds to an increase of $14 \%$ in VMT per capita.

To determine if the scenarios are significantly different we perform a two sample $t$-test for each FAZ. We find that most zones that contain a light rail station and thus, are affected by the density increase in the TOD scenario, do experience a significant decrease in VMT. They are depicted in the map in Figure 5 as shaded areas. FAZes with a decrease in the distribution mean of 5-10\% are shown in the lighter shading, while decreases larger than $10 \%$ are shown in the darker shade. The current and future light rail stations are shown as grey dots. 


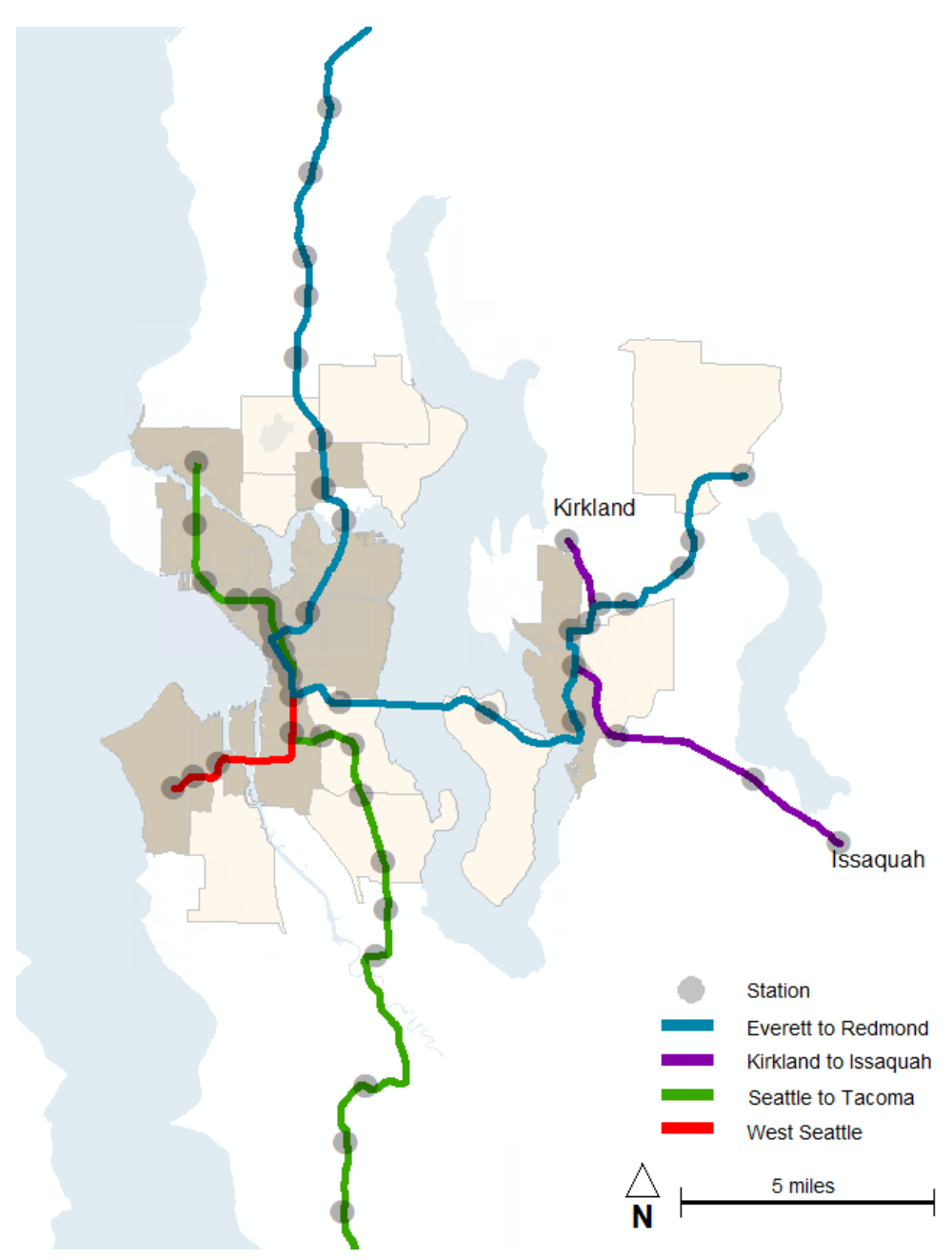

Figure 5: FAZes with significant decreases in mean VMT per capita. The lighter and darker shading represents decreases of $5-10 \%$ and $>10 \%$, respectively. Light rail stations are shown as grey dots. The map is zoomed so that it captures most of the light rail stations.

The $t$-test also reveals zones that show a significant increase in VMT. This could be explained by the land use changes introduced in the TOD scenario: Increasing density in the light rail buffers will yield a higher concentration of jobs in those areas which might be drawn away from rural places. This in turn will cause longer commutes for households that do not live in central areas.

Figure 6 shows the distribution of differences for zones with the largest difference between the distribution means. FAZes with the largest decrease in VMT are shown in the left panel while FAZes with the largest increase are shown in the right panel.

\subsubsection{Light Rail Boardings}

To present results of total boardings we select a light rail link between Kirkland and Issaquah shown in Figure 5 as the purple line. This link is planned to be built by 2041, thus very close to the analyzed 2040 year. It includes stations in highly populated areas (e.g. Bellevue) as well as stations in more rural places. The results are shown in Figure 7. It can be seen that some stations experience a significant increase in boardings (Bellevue TC and Main St.), while other stations might hardly be impacted by the zoning change. The graph also shows that the probability distributions add important information to the results. For example, for $S$ Bellevue P\&R station the point forecast might suggest there is up 


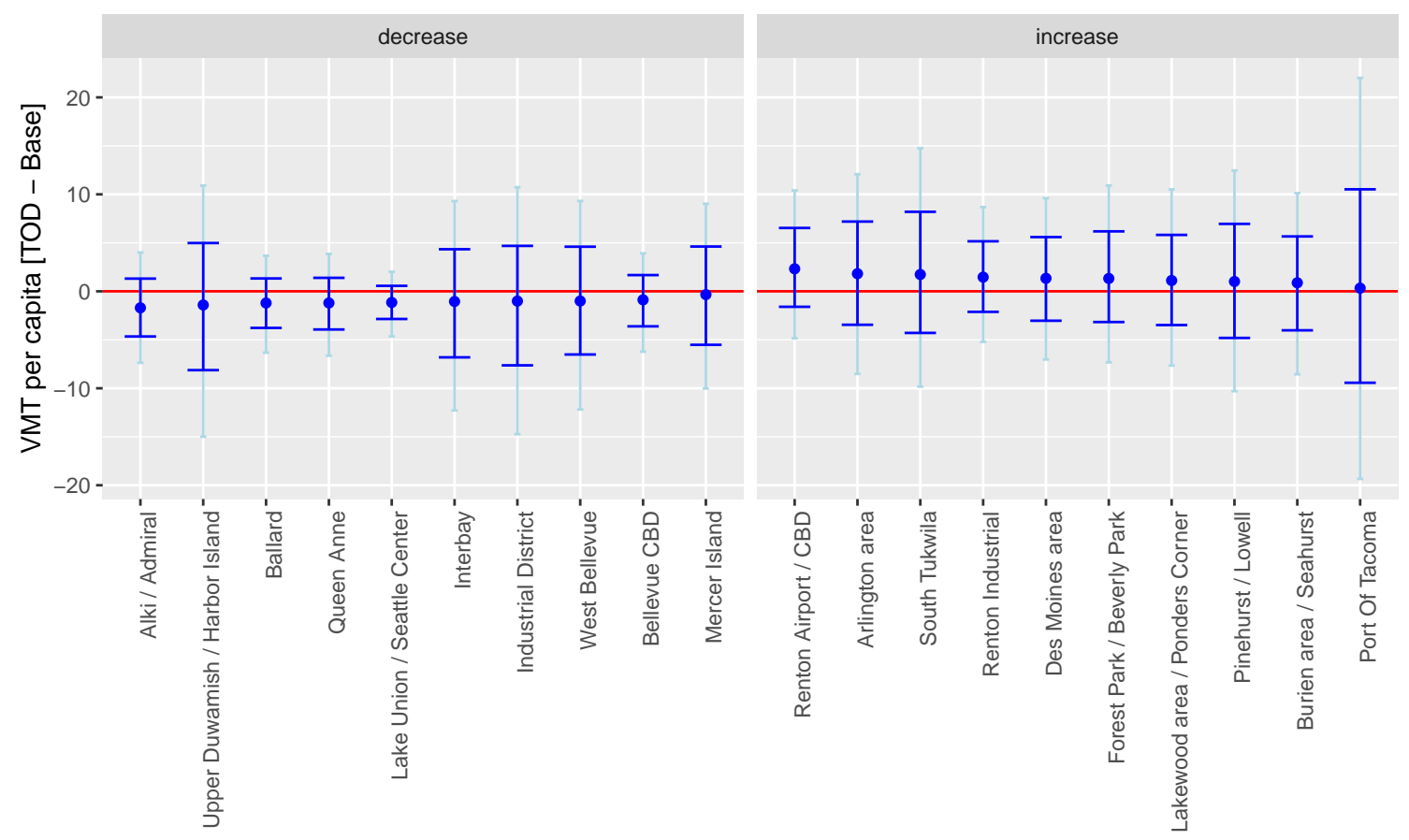

Figure 6: Predictive distributions of the differences in VMT per capita between the two scenarios (TOD - Base). The left panel contains ten FAZes with the largest negative difference between the distribution means, while the right panel contains ten FAZs with the largest positive difference. The whiskers and end lines depict the $50 \%$ and $80 \%$ probability intervals, respectively, while the dots show the medians of the distributions.

to $50 \%$ difference in boardings between the scenarios, depending on the runs analyzed. However, the overlapping distributions indicate that there is a non-zero probability that there will be no increase in the TOD scenario.

\subsubsection{Joint distribution}

In addition to the marginal distributions above, one can derive a joint distribution of multiple quantities of interest. Here, we join the marginal distribution of differences in VMT per capita (Figure 6) with an analogously constructed distribution of differences in boardings. Figure 8 shows the axes of such joint distribution for stations on the Kirkland-Issaquah light rail link. The horizontal lines contain $50 \%$ of the boardings difference distribution while the vertical lines contain $50 \%$ of the VMT per capita difference distribution and the lines cross in the respective medians. The joint distribution of the differences has an elliptical shape given by these axes, as shown for two of the stations. The graph suggests that four of the stations with a high probability of increased boardings also have a higher probability of decreased VMT per capita (bottom right quadrant). There is one station (Issaquah) where the VMT per capita has a higher probability of increase and at the same time a higher probability of decreased boardings (upper left quadrant), but a decrease in VMT per capita and an increase in boardings is also likely for this station. Even though the median boardings change of Bellevue TC (ellipse on the right) is about 2500 higher than the median change of its neighboring station, Main St. (ellipse in the middle), there is a positive probability that the change will be abound the same. Using distribution samples allows us to quantify probabilities of this type. Here, there is a $22 \%$ probability that the change at Main St. will be larger than the one at Bellevue TC. Similarly, there is a $30 \%$ chance 


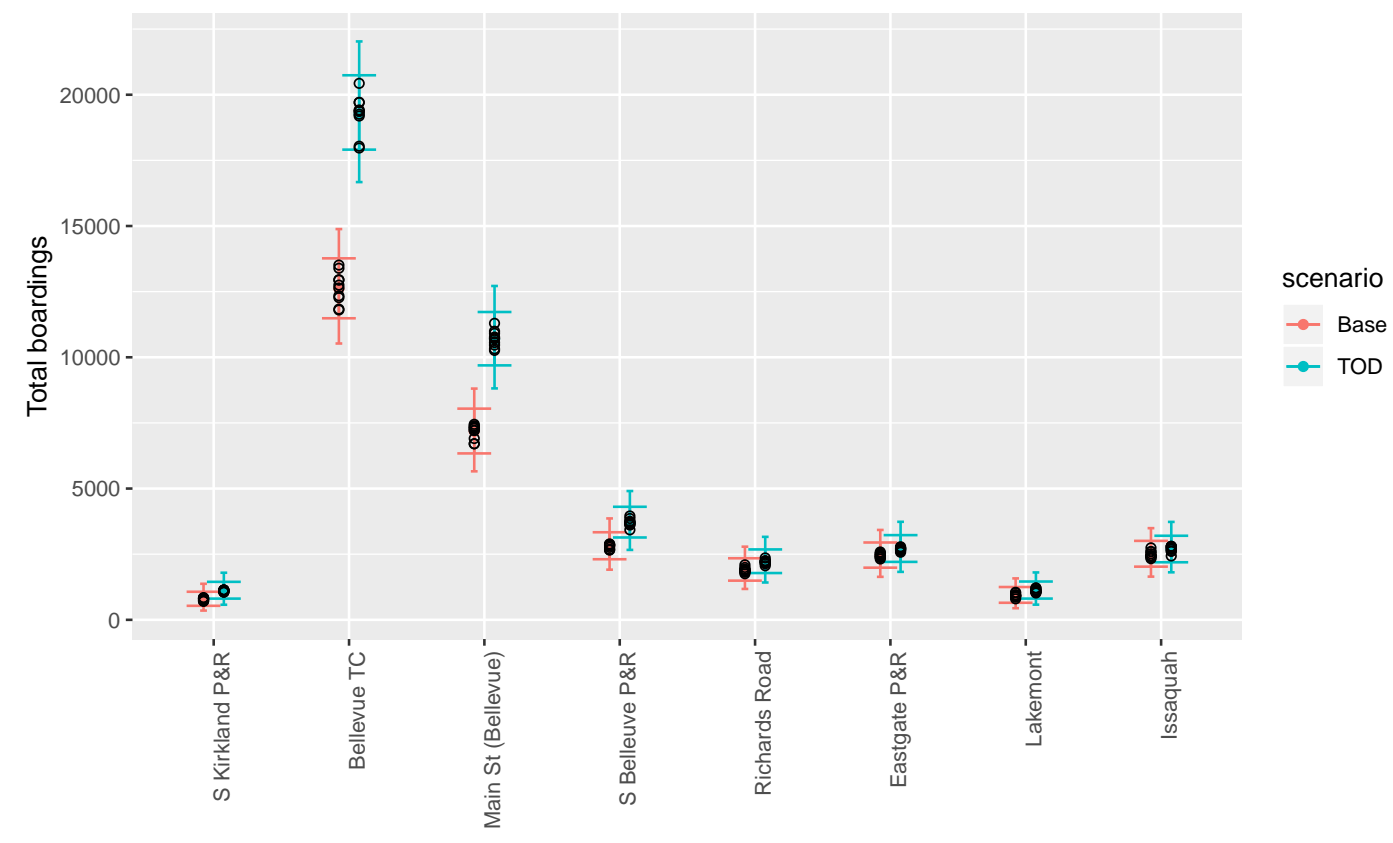

Figure 7: Predictive distributions of total boardings for the light rail link Kirkland-Issaquah for the two scenarios, base (red) and TOD (blue). The whiskers mark an area that contains $50 \%$ of the distribution, while the line ends mark the $80 \%$ probability intervals. The black dots show the point results from the model.

that both stations will have an increase in boardings of more than 2000 and at the same time have a decrease in VMT of more than half mile per capita.

\section{Conclusions and Future Work}

In this paper, we have extended the work on assessing uncertainty in land use models into the travel modeling area. In particular, to our best knowledge, propagating land use uncertainty assessed via a statistically grounded approach into a parcel level agent-based microsimulation travel model has not been done before. We used the land use model UrbanSim combined with methodology of Bayesian Melding to derive predictive posterior distributions of households in 2040 for each census block group. Sampling from these distributions and combining it with parcel-level information from multiple UrbanSim runs we set up multiple SoundCast runs that differ in their land use inputs consistent with the land use distributions. The quantities of interest in this study are vehicle miles traveled per capita and light rail boardings. In addition to the uncertainty originating in the different land use inputs, we added a direct source of uncertainty from the travel model, using a comparison between observed and forecasted VMT on the FAZ level and light rail boardings.

To demonstrate the practical application of the method we setup two scenarios, a base scenario and a scenario with increased density in the light rail buffers (TOD). We showed that very different conclusions could be reached if point forecasts are compared with no consideration of forecast accuracy. In one zone, without applying the presented method, the difference could be a $50 \%$ change for one set of runs or $0 \%$ change for another set of runs, while comparing distribution medians yields a more robust conclusion of a $14 \%$ change.

We plan to extend the study in several aspects, such as adding uncertainty on employment, adding sources of uncertainty about travel model input parameters, or relaxing the conditioning on external 


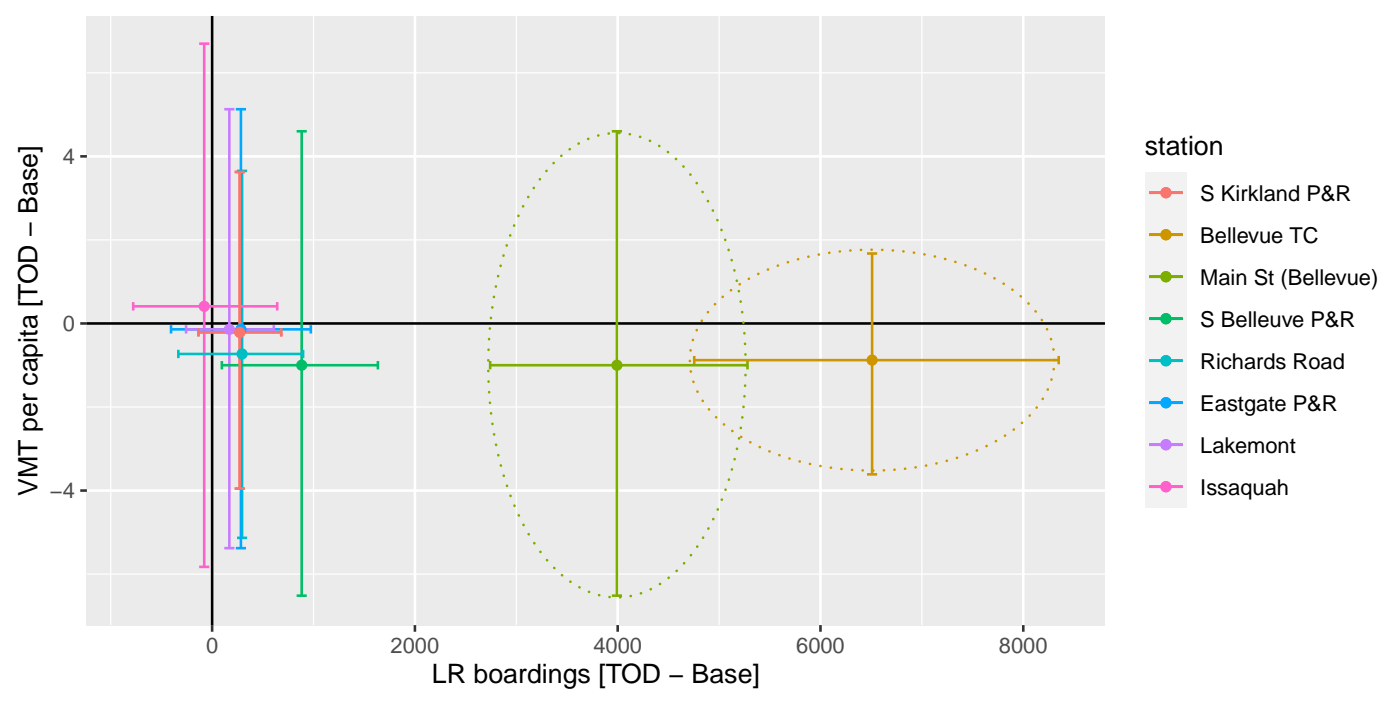

Figure 8: Joint predictive distributions of the differences in total boardings ( $x$ axis) and VMT per capita ( $y$ axis) between the two scenarios (TOD - Base). Colors represent stations on the light rail link KirklandIssaquah. The whiskers mark an area that contains $50 \%$ of the distribution. The elliptical $50 \%$ distribution bounds are shown for two stations.

control totals. We expect these additions to increase the spread between the simulated values. However, it remains to be seen if the distribution itself will become any wider. 


\section{References}

Bowman, J. and M. Bradley. 2014. SoundCast activity-based travel forecasting model for PSRC: Model system design. Puget Sound Regional Council. URL https://www.psrc.org/sites/default/ files/soundcastdesign2014.pdf.

Castiglione, J., M. Bradley, and J. Gliebe. 2015. Activity-based travel demand models: A primer. Second Strategic Highway Research Program, Transportation Research Board.

Clay, M. and R. Johnston. 2006. Multivariate uncertainty analysis of an integrated land use and transportation model: MEPLAN. Transportation Research Part D, 11(3):191 - 203.

Conway, M. W., A. Byrd, and M. van Eggermond. 2018. Accounting for uncertainty and variation in accessibility metrics for public transport sketch planning. Journal of Transport and Land Use, 11:541-558. URL https://doi.org/10.5198/jtlu.2018.1074/.

de Jong et al., G. 2005. Uncertainty in traffic forecasts: Literature review and new results for the Netherlands. Technical Report WR-268-AVV, Rand Corporation.

Duthie, J., A. Voruganti, K. M. Kockelman, and S. T. Waller. 2010. Uncertainty analysis and its impact on decision-making in an integrated transportation and gravity-based land use model. Journal of Urban Planning and Development, 136(4):294-302.

Felsenstein, D., K. Axhausen, and P. Waddell. 2010. Land use-transportation modeling with UrbanSim: Experiences and progress. Journal of Transport and Land Use, 3(2). URL https://doi.org/10. 5198/jtlu.v3i2.183.

Flyvbjerg, B. 2005. Measuring inaccuracy in travel demand forecasting: Methodological considerations regarding ramp up and sampling. Transportation Research Part A, 39:522-530.

Flyvbjerg, B., N. Bruzelius, and W. Rothengatter. 2003. Megaprojects and Risk. New York: Cambridge University Press.

Flyvbjerg, B., M. K. S. Holm, and S. L. Buhl. 2005. How (in)accurate are demand forecasts in public works projects? The case of transportation. Journal of the American Planning Association, 71(2):131-146.

Gibb, J. and J. Bowman. 2007. Convergence of an activity-based travel model system to equilibrium: Experimental designs and findings. In 11th National Transportation Planning Applications Conference.

Hartgen, D. T. 2013. Hubris or humility? Accuracy issues for the next 50 years of travel demand modeling. Transportation, 40:1133-1157. URL https://doi.org/10.1007/s11116-013-9497-y.

Hugosson, M. B. 2005. Quantifying uncertainties in a national forecasting model. Transportation Research Part A, 39:531-547.

Kockelman, K. M. 2002. Uncertainty propagation in an integrated land use-transport modeling framework: Output variation via UrbanSim. Transportation Research Record, (1805):128-135.

Kockelman, K. M. 2003. Propagation of uncertainty in transportation-land use models: An investigation of DRAM-EMPAL and UTPP predictions in Austin, Texas. Transportation Research Record, (1831):219-229.

Pontius Jr, R. G. and J. Spencer. 2005. Uncertainty in extrapolations of predictive land-change models. Environment and Planning B: Planning and Design, 32:211-230.

Poole, D. and A. E. Raftery. 2000. Inference for deterministic simulation models: The Bayesian melding approach. Journal of the American Statistical Association, 95(452):1244-1255.

Pradhan, A. and K. M. Kockelman. 2002. Uncertainty propagation in an integrated land usetransportation modeling framework - output variation via UrbanSim. Transportation Research Record: Journal of the Transportation Research Board, 1805(1):140-150.

Puget Sound Regional Council. 2018. The Regional Transportation Plan. URL https://www.psrc. org/sites/default/files/rtp-may2018.pdf.

Raftery, A. E., G. H. Givens, and J. E. Zeh. 1992. Inference from a deterministic population dynamics model about bowhead whale, balaena mysticetus, replacement yield. Technical Report 232, Depart- 
ment of Statistics, University of Washington, Seattle, Wash.

Raftery, A. E., G. H. Givens, and J. E. Zeh. 1995. Inference from a deterministic population dynamics model for bowhead whales. Journal of the American Statistical Association, 90(430):402-416.

Rodier, C. J. 2005. Verifying the Accuracy of Land Use Models Used in Transportation and Air Quality Planning: A Case Study in the Sacramento, California Region. Institute of Transportation Studies, University of California Davis. URL transweb.sjsu.edu/MTIportal/research/publications/ summary/0502.html.

Salling, K. B. and D. Banister. 2009. Assessment of large transport infrastructure projects: The CBADK model. Transportation Research Part A, 43:800-813.

Ševčíková, H., A. Raftery, and P. Waddell. 2007. Assessing uncertainty in urban simulations using Bayesian melding. Transportation Research B, 41:652-669.

Ševčíková, H., A. Raftery, and P. Waddell. 2011. Uncertain benefits: Application of Bayesian melding to the Alaskan Way Viaduct in Seattle. Transportation Research A, 45:540-553.

Ševčíková, H., M. Simonson, and M. Jensen. 2015. Assessing and integrating uncertainty into land use forecasting. Journal of Transport and Land Use, 8(3):5-70. URL https://doi.org/10.5198/jtlu. 2015.614.

Sumalee, A., P. Luathep, W. H. K. Lam, and R. D. Connors. 2009. Evaluation and design of transport network capacity under demand uncertainty. Network Modeling, 1:17-28.

Tayman, J. 1996. The accuracy of small-area population forecasts based on a spatial interaction land use modeling system. Journal of the American Planning Association, 62(1):85-98. doi: $10.1080 / 01944369608975672$.

Tayman, J. 2009. Uncertainty in Socioeconomic Modeling Systems: Assessment and Recommendations. Maricopa Association of Governments, Phoenix, AZ. URL http://www.azmag.gov/Documents/ IS_2009-06-05_Socioeconomic-Modeling_Uncertainty-White-Paper.pdf.

Tayman, J. 2011. Assessing uncertainty in small area forecasts: State of the practice and implementation strategy. Population Research and Policy Review, 30:781-800. doi: 10.1007/s11113-0119210-9.

Waddell, P. 2002. UrbanSim: Modeling urban development for land use, transportation, and environmental planning. Journal of the American Planning Association, 68(3):297-314.

Waddell, P. 2007. Accessibility and residential location: the interaction of workplace, housing tenure, residential mobiltity and location choice. In J. Preston, F. Pagliara, and D. Simmonds, eds., Modelling Residential Location Choice. Ashgate. In press.

Waddell, P. 2010. Modeling residential location in urbansim. In J. Preston, F. Pagliara, and D. Simmonds, eds., Modelling Residential Location Choice, pp. 165-180. Springer.

Waddell, P. 2011. Integrated land use and transportation planning and modeling: Addressing challenges in research and practice. Transport Reviews, 31(2):209-229.

Waddell, P., H. Ševčíková, D. Socha, E. Miller, and K. Nagel. 2005. Opus: An Open Platform for Urban Simulation. Presented at the Computers in Urban Planning and Urban Management Conference, London.

Waddell, P., L. Wang, H. Ševčíková, and A. Borning. 2015. Agile modeling: Adapting UrbanSim to the European context using the Open Platform for Urban Simulation. In M. Bierlaire, A. de Palma, R. Hurtubia, and P. Waddell, eds., Integrated Transport and Land Use Modelingfor Sustainable Cities, chapter 16. Routledge.

Wang, G. and K. M. Kockelman. 2018. Three methods for anticipating and understanding uncertainty of outputs from transportation and land use models. Transportation Research Record, 2672:12-22.

Yang, L. and Y. Guo. 2019. Combining pre- and post-model information in the uncertainty quantification of non-deterministic models using an extended Bayesian melding approach. Information Sciences, 502:146-163. URL https://doi.org/10.1016/j.ins.2019.06.029.

Zhao, Y. and K. M. Kockelman. 2002. The propagation of uncertainty through travel demand models: An exploratory analysis. Annals of Regional Science, 36(2):145-163. 